plates. CIK cells in G-Rex $6{ }^{\circledR}$ were split only once at day 7 to reduce cell density, whereas the number of CIK cells culterd in G-Rex ${ }^{\circledR} 6 \mathrm{M}$ was not adjusted. In both culture conditions, fresh IL-2 was provided every 3-4 days. We compared these two culture protocols with the culture in standard flasks. Phenotype was analyzed by flow cytometry and cytotoxicity was assessed against several tumor cell lines by calcein-release assay.

Results CIK cells cultured in G-Rex $6{ }^{\circledR}$ well plates showed an outstanding cell expansion compared to G-Rex ${ }^{\circledR} 6 \mathrm{M}$ well plates or standard culture flasks, with a 400-fold expansion and a mean of $10^{9}$ total cells obtained per single well in 14 days, starting from just $2.5 \times 10^{6}$ cells per well. Moreover, the cultures in G-Rex $6{ }^{\circledR}$ were characterized by an higher percentage of $\mathrm{CD}^{+}{ }^{+} \mathrm{CD} 56^{+}$cells, as compared to $\mathrm{G}-\mathrm{Rex}{ }^{\circledR} 6 \mathrm{M}$ or standard culture flasks. Cells cultured in all devices had a comparable expression of NKG2D, NKp30, NKp44, 2B4 receptors. Importantly, CIK cells expanded in G-Rex ${ }^{\circledR} 6$ were as cytotoxic as cells expanded in standard culture flasks. Conversely, CIK cells cultured in G-Rex ${ }^{\circledR} 6 \mathrm{M}$ showed a remarkable reduction of cytotoxicity against tumor cell targets, thus suggesting that cell density during expansion could affect CIK cell activity.

Conclusions We propose a GMP-compliant protocol for robust large-scale production of CIK cells. G-Rex ${ }^{\circledR}$ system allows to obtain large amounts of CIK cells highly enriched in the $\mathrm{CD}^{+} \mathrm{CD} 6^{+}$subset and endowed with high cytotoxic activity; this can be accomplished with just a single cell culture split at day 7 , which dramatically reduces the culture manipulation as compared to the standard culture flasks. Notably, this strategy can be further and easily scalable to produce CIK cells for clinical immunotherapy applications.

Disclosure Information A. Ventura: None. P. Palmerini: None. A. Dalla Pietà: None. R. Sommaggio: None. G. Astori: None. K. Chieregato: None. M. Tisi: None. C. Visco: None. O. Perbellini: None. M. Ruggeri: None. E. Cappuzzello: None. A. Rosato: None.

\section{P09.14 BLOCKING COUNTERREGULATION OF UNFOLDED PROTEIN RESPONSE BY TARGETED PROTEIN SYNTHESIS INHIBITION PRODUCES HIGHLY SYNERGISTIC CELL DEATH IN SEVERAL CANCER ENTITIES}

F Gsottberger*, C Meier, S Petkovic, L Mellenthin, M Krumbholz, M Metzler, A Mackensen, F Müller. University Hospital of Erlangen, Erlangen, Germany

\subsection{6/jitc-2020-ITOC7.114}

Background Because tumor cells have high proliferation rates the demand for energy on the one hand and proteins on the other hand is high. In line, protein folding machinery of the ER is heavily used. 2-Deoxyglucose (2-DG) not only blocks energy synthesis by inhibiting glycolysis but also blocks synthesis of mannosyl leading to impaired N-linked glycosylation, accumulation of misfolded proteins, and increased unfolded protein response (UPR). However, due to compensatory events, UPR-induced apoptosis is hampered. Therefore, we combined 2-DG with targeted protein synthesis inhibition by immunotoxins, consisting of an antibody and pseudomonas exotoxin, to enhance UPR mediated cell death.

Materials and Methods Established cell lines and patientderived B-ALL samples were treated in vitro with various protein synthesis inhibitors and UPR-inducers. Drug synergy was determined mathematically as fold-increase over additivity.
Biochemical studies were performed using western blots. In vivo enhancement was tested using systemic xenograft models. Results The combination of Moxetumomab and 2-DG achieved a two to nine-fold synergy in vitro. Synergy was abrogated by the addition of Mannose suggesting UPR as cause of synergistic cell death. Similarly, Moxetumomab enhanced UPR-inducers Bortezomib and tunicamycin and protein synthesis inhibition by cycloheximide and puromycin enhanced 2-DG suggesting a conserved mechanism. Using HB21, an immunotoxin targeting human transferrin-receptor, breast cancer, hepatocellular carcinoma, and glioblastoma were sensitized to 2-DG induced cell death. Biochemically, 2-DG increased XBP-1-cleavage, expression of pro-apoptotic CHOP and of anti-apoptotic BIP. Moxetumomab, however, blocked the upregulation of BIP while maintaining CHOP correlating with synergistic increase in PARP-cleavage and apoptosis. In two systemic mouse models, bone marrow (BM) lymphoma infiltration was not reduced by 2-DG or tunicamycin alone but was reduced after treatment with Moxetumomab alone by 5 -fold in the JeKo- 1 and by 16 -fold in the Ramos model, respectively. The combination of Moxetumomab and 2-DG achieved a three-fold synergy in the JeKo-1 model and achieved MRD-negative BM status in the Ramos model. Against patient-derived B-ALL of the Burkitt's type, 2-DG and Moxetumomab were up to 5 -fold more active in vitro and up to 7 -fold more active in mouse xenografts in vivo.

Conclusions Cell death after persisting unfolded protein response is synergistically enhanced by tumor-cell specific inhibition of protein synthesis against four distinct tumor entities at physiologically achievable concentrations. Our approach of immunotoxin-induced targeted protein synthesis inhibition opens a novel, so far undescribed therapeutic window which may warrant clinical evaluation.

Disclosure Information F. Gsottberger: None. C. Meier: None. S. Petkovic: None. L. Mellenthin: None. M. Krumbholz: None. M. Metzler: None. A. Mackensen: None. F. Müller: None.

\section{P09.15 TARGETING THE STROMA TO ENHANCE EFFECTOR MEMORY T CELL INFILTRATION AND ANTI-TUMOR RESPONSE TO ANTI-PD1 ANTIBODY IN PANCREATIC DUCTAL ADENOCARCINOMA}

A Osipov*, L Zheng. Johns Hopkins University, Baltimore, MD, USA

\subsection{6/jitc-2020-ITOC7.115}

Background Pancreatic ductal adenocarcinoma (PDAC) is resistant to immune checkpoint inhibition. One of the major resistance mechanisms is attributed to myeloid cells as an immunosuppressive element within the stroma of PDAC. It has been reported that focal adhesion kinase inhibitor (FAKi) can suppress immunosuppressive myeloid cells such as tumor associated macrophages (TAMs) and myeloid derived suppressor cells (MDSC), consequently sensitizing tumor to anti-PD1 antibody in mouse models of PDAC. Our group has previously shown in a murine model that targeting the stroma via PEGylated recombinant human hyaluronidase (PEGPH20) enhanced the anti-tumor activity of the whole cell vaccine (GVAX) by targeting CXCR4-expressing myeloid cells and led to an increase in infiltration of CCR7- effector memory $\mathrm{T}$ cell subsets. Here, we evaluate the hypothesis that FAK expressing myeloid cell subsets modulate $\mathrm{T}$ cell infiltration in human 
PDAC and FAKi can synergize with PEGPH20 by targeting myeloid cells in PDAC.

Material and Methods Resected human PDAC tissue specimens treated with GVAX and anti-PD1 therapy was used to assess FAK expression in myeloid cell subsets and its impact on $\mathrm{T}$ cell infiltration. A sequential staining and stripping multiplex IHC technique that incorporates 28 myeloid and lymphoid biomarkers, as well as phosphorylated FAK (pFAK) combined with computational image processing was used to assess myeloid cell populations, $\mathrm{T}$ cell infiltration and FAK expression.

An established murine model of metastatic PDAC treated with and without anti-PD1 therapy was used to assess the synergy and immune-modulating effect of FAKi and stromal degradation of hyaluronan via PEGPH20.

Results In human PDAC, FAK is widely expressed in TAMs and neutrophils. Increased FAK expression is associated with increased CXCR4 expression. Lower pFAK density in neutrophils and M2 TAMs, but not lower pFAK density in M1 TAMs, is associated with higher CD8 $+\mathrm{T}$ cell infiltration.

FAKi and combination of FAKi with anti-PD1 extends survival in the mouse metastasis model of PDAC. Adding PEGPH20 to FAKi and anti-PD1 antibody significantly prolonged survival in this model. Comparing to the combination of FAKi and anti-PD1 antibody, adding PEGPH20 significantly decreased the number of CXCR4-expressing myeloid cells in the tumor microenvironment (TME) of PDAC and consequently led to an increase in the amount of CCR7+ central memory $\mathrm{T}$ cells. Additionally, the amount of G-MDSCs, inflammatory resident monocytes and PDL1 expressing myeloid cells in the TME of PDAC, was also decreased in PDAC treated with the triple combination of PEGPH20, FAKi and anti-PD1 antibody compared to FAKi and anti-PD1 antibody.

Conclusion FAK is widely expressed in myeloid cell populations, directly correlated with CXCR4 expression and decreased FAK expression in a myeloid (M2 TAMs, neutrophil) inflamed stroma is associated with infiltration of effector CD8 $\mathrm{T}$ cells in human PDAC. Stromal degradation of hyaluronan via PEGPH20 combined with FAKi and anti-PD1 antibody further depletes immunosuppressive cells in the TME including G-MDSCs, inflammatory resident monocytes and PDL1 expressing myeloid cells and appears to target the CXCR4 pathway through PEGPH20. These findings support testing the combination of FAKi and anti-PD1 antibody with agents targeting CXCR4 directly or indirectly by PEGPH20 in human PDAC.

Disclosure Information A. Osipov: None. L. Zheng: None. 